\title{
Influência do intervalo de recuperação entre alongamento e treinamento de força
}

\section{Influence of rest interval between stretching and resistance training}

\author{
Gabriel Andrade Pazi; Thalita Leite; ; Marianna de Freitas Maia'; Andressa Ferreira Lima4; \\ Poliana Pires Coelho4; Roberto Simão3; Humberto Miranda ${ }^{3}$ \\ ${ }^{1}$ Mestrandos do Programa Stricto Sensu em Educação Física - Escola de Educação Física e Desportos - Universidade Federal do Rio de \\ Janeiro - UFRJ. Rio de Janeiro, RJ - Brasil. \\ ${ }_{2}$ Professora, Mestre em Educação Física - Escola de Educação Física e Desportos - Universidade Federal do Rio de Janeiro - UFRJ. Rio \\ de Janeiro, RJ - Brasil. \\ Professores Doutores e Membros do Laboratório de Treinamento de Força da Escola de Educação Física e Desportos - Universidade \\ Federal do Rio de Janeiro - UFRJ. Rio de Janeiro, RJ - Brasil. \\ ${ }^{4}$ Professoras, Graduadas em Educação Física - Programa de Pós-Graduação Lato Sensu em Musculação e Treinamento de Força - \\ Universidade Gama Filho - UGF. Rio de Janeiro, RJ - Brasil. \\ Endereço para correspondência \\ Gabriel Andrade Paz \\ Av. Carlos Chagas Filho, Cidade Universitária \\ 21941-590 - Rio de Janeiro - RJ [Brasil] \\ gabriel.andrade.paz@gmail.com
}

\section{Resumo}

Objetivo: Investigar o efeito de diferentes intervalos de recuperação entre alongamento estático passivo para o quadríceps no desempenho de repetições máximas nos exercícios leg press $45^{\circ}$ e cadeira extensora. Métodos: Dezesseis mulheres treinadas (22,2 $\pm 3,6$ anos; $57,2 \pm 3,42 \mathrm{~kg} ; 1,66 \pm 32,3 \mathrm{~cm})$ executaram quatro sequências de treinamento em dias não consecutivos. PSI: iniciava pelo alongamento estático, seguido pelos exercícios resistidos sem intervalo; P10: 10 minutos de intervalo entre o alongamento e os exercícios resistidos; P20: 20 minutos de intervalo entre o alongamento e os exercícios resistidos; PSA: os exercícios resistidos foram realizados sem alongamento prévio. Resultados: Houve redução significativa no total de repetições máximas realizadas no PSI, P10 e P20, comparado ao PSA em ambos os exercícios, exceto para o P20 na cadeira extensora, no qual não foi observada diferença para com o PSA. Conclusão: Verificou-se que o intervalo de 20 minutos possibilitou total recuperação do desempenho muscular comparado ao PSA na cadeira extensora.

Descritores: Exercício; Exercício de alongamento muscular; Força muscular; Treinamento de resistência.

\begin{abstract}
Objective: To investigate the effect of different rest intervals between passive static stretching for quadriceps muscles and the performance of repetition maximum at $45^{\circ} \mathrm{leg}$ press and leg extension exercises. Methods: Sixteen trained women $(22.2 \pm 3.6$ years, $57.2 \pm 3.42 \mathrm{~kg}, 1.66 \pm 32.3 \mathrm{~cm})$ executed four sequences training on nonconsecutive days. PWI: initiated by static stretching followed by resistance exercises; P10: 10 minutes between static stretching and resistance exercises; P20: 20 minutes between static stretching and resistance exercises; PWS: the resistance exercises were performed without previous stretching. Results: A significant reduction in total maximum repetitions completed in PWI, P10 and P20 compared to PWS in the exercise of leg extension and leg press, except at P20 in the leg extension which showed no difference for the PWS. Conclusion: The 20 minute rest interval between static stretching and RT allowed a completely recovery of repetition performance for leg extension compared to PWS.
\end{abstract}

Key words: Exercise; Muscle stretching exercise, Muscle strength; Resistance training. 


\section{Introdução}

O treinamento de força (TF) desempenha um papel fundamental nos programas de atividade física e tem sido recomendado por diversas organizações de saúde ${ }^{1,2}$. De acordo com o posicionamento do American College of Sports Medicine $(\mathrm{ACSM})^{3}$, a prescrição do $\mathrm{TF}$ aliado ao treinamento de flexibilidade é recomendada visando a manutenção de força, resistência muscular e flexibilidade dos principais grupos musculares.

Neste sentido, evidências relacionadas aos efeitos que cada variável pode gerar em uma sessão de treinamento são importantes para maximizar os resultados e auxiliar o indivíduo a alcançar os seus objetivos. Segundo Ribeiro ${ }^{4}$, a amplitude articular pode exercer influência limitando o desenvolvimento de contrações voluntárias máximas, aumentando, assim, o gasto energético e tornando mais difícil o trabalho a ser realizado. Viveiros e Simão ${ }^{5}$ afirmam que os exercícios de alongamento podem manter ou aumentar a flexibilidade, sendo, por isso, aplicados para prevenir encurtamentos teciduais e otimizar o desempenho muscular, contribuindo também para o TF e potência muscular.

Entre os métodos utilizados para o treinamento da flexibilidade, o alongamento estático passivo (AEP) é o aplicado com maior frequência pela segurança e facilidade de seu uso, sendo caracterizado pelo alcance de uma amplitude de movimento articular lentamente até o ponto de desconforto ou dor ${ }^{6}$. Entretanto, estudos prévios indicam que a aplicação de AEP pode promover efeito agudo deletério sobre a força muscular. Silva et al. ${ }^{7}$ referiram diminuição significativa na força isométrica máxima após exercícios de AEP nos flexores do punho, com duração de 30 segundos, em grupo de homens e mulheres treinadas. Endlich et al. ${ }^{8}$ verificaram redução significativa na produção de força durante o teste de dez repetições máximas (RM) nos exercícios supino e leg press $45^{\circ}$, após sessões de AEP, de 8 e 16 minutos, em adolescentes treinados em TF. Resultados semelhantes foram encontrados por
Gurjão et al. ${ }^{9}$, que observaram redução significativa no sinal eletromiográfico e na produção de força dos extensores do joelho após aplicação de AEP em mulheres idosas. Há de se considerar, que em diversos trabalhos foram realizadas sessões de TF imediatamente após a aplicação do AEP. Neste contexto, destaca-se que, até o presente momento, não há consenso quanto ao efeito de diferentes intervalos de recuperação entre os exercícios de AEP e sessões de TF sobre o desempenho muscular. Egan et al. ${ }^{10}$ não observaram alterações no pico de torque e a potência média durante a contração voluntária máxima dos extensores de joelho em jogadoras de basquete, quando adotaram intervalos de 5 , 15, 30 e 45 minutos entre o AEP e sessões de TF. Todavia, Fowles, Sale e MacDougall ${ }^{11}$ constataram que a contração voluntária máxima (CVM) no tríceps sural reduziu em $28 \%$, após AEP, e verificaram diminuição de $9 \%$, depois de uma hora. Mcbridge, Deane e Nimphius ${ }^{12}$ mencionaram redução significativa na CVM 1, 2, 8 e 16 minutos após AEP.

Destaca-se que a manutenção dos níveis de flexibilidade é fundamental em programas de exercícios visando a qualidade de vida e a saúde ${ }^{3}$; entretanto, estudos prévios indicam que a aplicação de AEP pode promover efeito agudo deletério sobre a força muscular. Dessa forma, é importante evitar efeitos prejudiciais à força muscular em sessões de TF após o treino de flexibilidade ${ }^{13}$. Adicionalmente, o tempo despendido no treinamento pode ser otimizado quando o treino concorrente de flexibilidade e força é aplicado em sequência. Assim, dados associados a intervalos de recuperação que sejam adequados para a manutenção do desempenho de força muscular, após exercícios de AEP, podem ser úteis e aperfeiçoar os resultados de praticantes e profissionais da área de treinamento, possivelmente orientando a prescrição e elaboração de programas de TF.

Portanto, objetivo-se neste estudo investigar o efeito de diferentes intervalos de recuperação entre exercícios de alongamento estático passivo para o quadríceps, e o desempenho de 
repetições máximas nos exercícios leg press e cadeira extensora em mulheres treinadas em TF. Uma das hipóteses neste trabalho está associada a melhora no desempenho de repetições máximas, após AEP, quando adotados intervalos de recuperações superiores a dez minutos, em comparação ao TF realizado imediatamente após exercícios de AEP.

\section{Material e métodos}

\section{Amostra}

Participaram nesta pesquisa 16 mulheres jovens $(22,2 \pm 3,6$ anos; $57,2 \pm 3,42 \mathrm{~kg} ; 1,66 \pm 32,3$ $\mathrm{cm})$ com experiência prévia em TF $(2 \pm 1,2$ anos $)$ e selecionadas de forma não probabilística. As voluntárias foram familiarizadas com os exercícios utilizados. Com a finalidade de satisfazer os critérios de inclusão propostos, todas deveriam apresentar as seguintes características: (a) possuir experiência em TF há pelo menos seis meses antes do início deste trabalho; (b) não executar nenhum outro tipo de atividade física regular durante o estudo; (c) não apresentar nenhuma limitação funcional para o TF ou para o desempenho dos testes envolvidos; (d) não ter nenhuma limitação médica que pudesse influenciar no programa de treinamento; (e) apresentar PAR-Q negativo. Antes de iniciar a coleta de dados, todas responderam ao questionário PAR-Q e assinaram um Termo de Consentimento Livre e Esclarecido, que explica os procedimentos dos testes e do treinamento realizados durante o estudo, conforme a Resolução 196/96 do Conselho Nacional de Saúde para experimentos com humanos. O Projeto de Pesquisa foi aprovado pelo Comitê de Ética da Universidade Gama Filho, sob o número de protocolo 043/2010.

\section{Teste de 1 Repetição Móxima (lRM)}

A coleta de dados foi efetuada em seis dias com intervalo de 48 horas entre eles. No primeiro dia, realizou-se o teste de 1RM no leg press (LP) e na cadeira extensora (CE). No segundo dia, após
48-72 horas de intervalo, os testes foram repetidos a fim de verificar a reprodutibilidade das cargas. $\mathrm{O}$ teste de $1 \mathrm{RM}$ foi adotado a partir do protocolo de medidas de força muscular de forma não invasiva. O procedimento do teste tinha o propósito de obter a carga máxima nos exercícios cadeira extensora (CE) e leg press $45^{\circ}(\mathrm{LP})^{14}$. Durante o protocolo, adotaram-se os seguintes procedimentos $^{15}$ : (a) foram fornecidas às participantes instruções padronizadas sobre os procedimentos de teste antes dos testes; (b) deram-se orientações referente à técnica de execução dos exercícios; (c) promoveram-se estímulos verbais a todas as jovens durante os testes; (d) determinouse o peso de todas as placas e barras utilizadas por meio de uma balança de precisão. Durante os testes, um avaliador experiente estava atento à posição adotada pelo praticante no momento da medida, pois pequenas variações no posicionamento das articulações envolvidas na ação poderiam recrutar outros músculos, possibilitando interpretações errôneas dos escores obtidos. O 1RM foi determinado por no máximo três tentativas para cada exercício, com um intervalo de dois a cinco minutos entre elas. Depois que a carga de $1 \mathrm{RM}$ de um exercício era determinada, um intervalo de, no mínimo, dez minutos era concedido antes da determinação de 1RM do seguinte.

Após a obtenção das cargas máximas no teste de $1 \mathrm{RM}$, as participantes descansaram por 48-72 horas e foram reavaliadas para obtenção da reprodutibilidade do teste. A maior carga atingida nos dias de teste foi considerada o 1RM pré-treino. A fim de não interferir nos resultados de reprodutibilidade, nenhum exercício foi permitido nas 48 horas entre os testes de 1RM.

\section{Protocolo de alongamento estático passivo}

De acordo com as recomendações do $\mathrm{ACSM}^{3}, 10$ a 30 segundos de tensão são recomendados para a maioria dos adultos, resultando em um volume total de 60 segundos para cada exercício de alongamento muscular, realizados de 2 a 4 séries. Assim, foram realizadas três séries de 
AEP, sustentando a posição por 20 segundos em cada série, até que o ponto de leve desconforto fosse encontrado. $\mathrm{O}$ alongamento foi realizado unilateralmente, alternando os lados sem pausa entre as séries. Para tal, os indivíduos foram posicionados em pé com os pés paralelos e os joelhos semiflexionados, usando o espaldar como apoio, flexionando uma das pernas em direção ao glúteo (Figura 1).

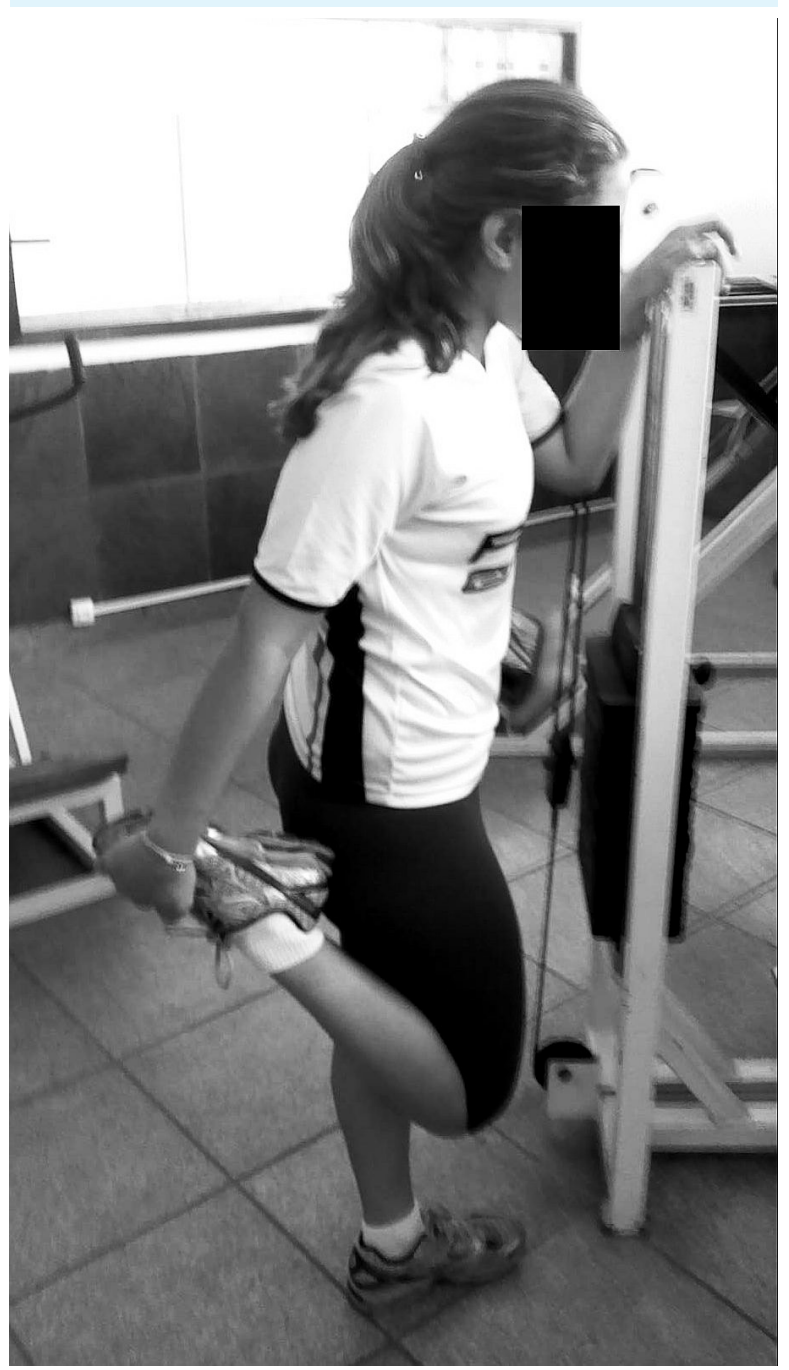

Figura 1: Alongamento estático passivo para o quadríceps

\section{Protocolo experimental}

Os exercícios de LP e CE foram selecionados devido à sua disseminação em centros de treinamento e facilidade de execução. É dada, a seguir, uma breve descrição da posição inicial (PI) e da fase concêntrica (FC) dos exercícios:

- LP: PI - a voluntária posicionou-se sentada no aparelho mantendo $90^{\circ}$ de flexão de quadril e joelhos, bem como dorsiflexão de $30^{\circ}$ no tornozelo. As mãos seguravam o apoio manual posicionado na linha orbital ao lado da cabeça. FC - realizou-se a extensão completa do quadril e joelhos, e flexão plantar. Durante a fase excêntrica a participante controlou o movimento de flexão do quadril e joelho, e flexão plantar até retornar para a PI. Não houve controle de cadência durante os exercícios.

- CE: PI - a jovem posicionou-se sentada, quadril e joelhos em flexão em aproximadamente $90^{\circ}$. FC - realizou-se a extensão completa dos joelhos mantendo o tornozelo na posição neutra. Durante a fase excêntrica a voluntária controlou o movimento de flexão dos joelhos até retornar para a PI.

Após a obtenção da carga de 1RM para cada exercício, as voluntárias executaram os protocolos experimentais pela entrada alternada, seguindo o quadrado latino com 48 horas de intervalo entre as sessões de testes. Todos os exercícios do TF foram realizados em três séries, com $85 \%$ de $1 \mathrm{RM}$, até a falha concêntrica no LP e na $\mathrm{CE}$, adotando-se dois minutos de intervalo de recuperação entre as séries e os exercícios.

- PSI: o protocolo sem intervalo iniciava pelo AEP para o quadríceps seguido imediatamente pelos exercícios LP e CE. P10: neste protocolo adotou-se dez minutos de intervalo entre o AEP para o quadríceps e séries múltiplas (três) nos exercícios LP e CE, respectivamente. P20: determinaram-se 20 minutos de intervalo entre o AEP para o quadríceps e séries múltiplas nos exercícios LP e CE. PSA, neste protocolo, foram realizadas três séries nos exercícios $\mathrm{LP}$ e CE (Figura 2). 


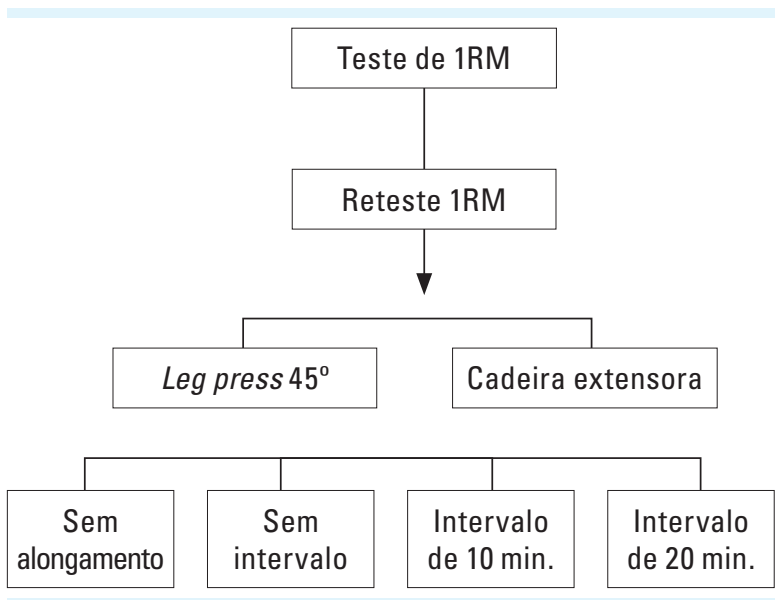

Figura 2: Design metodológico do estudo

Durante as sessões de exercícios, as participantes foram verbalmente incentivadas a executar todas as séries até a falha concêntrica, preservando as amplitudes pré-determinadas. As mesmas definições da execução foram aplicadas durante o teste de 1RM para definir uma repetição como validada. A velocidade com a qual as repetições eram executadas não foi controlada. O número total de repetições por série em cada exercício foi registrado.

\section{Tratamento estatístico}

Todos os dados foram apresentados com média \pm desvio-padrão (DP). A análise estatística foi realizada inicialmente utilizando o teste de normalidade de Shapiro-Wilk e teste de homocedasticidade (critério Bartlett). Todas as variáveis apresentaram distribuição normal e homocedasticidade. O coeficiente de correlação intraclasse $\left(\mathrm{CCI}=\left(\mathrm{MS}_{\mathrm{b}}-\mathrm{MS}_{\mathrm{w}}\right) /\left[\mathrm{MS}_{\mathrm{b}}+(\mathrm{k}-1) \mathrm{MS}_{\mathrm{w}}\right)\right.$ foi calculado para verificar a reprodutibilidade do teste e reteste de 1RM nos exercícios CE e LP. Foi utilizada uma análise de variância (Anova one-way) para medidas repetidas para comparar o somatório das repetições máximas realizadas nas três séries em cada exercício e, quando necessário, o post hoc de LSD foi usado para identificação das diferenças. Em todos os casos, adotaram-se como nível de significância estatística $p$ $\leq 0,05$. O software SPSS, versão 20.0, foi utilizado em todas as análises estatísticas.

\section{Resultados}

Os CCI obtidos no teste e reteste de 1RM foram de 0,91 e 0,98 nos exercícios de CE e LP, respectivamente. Os somatórios do número de repetições máximas realizadas nas três séries nos exercícios LP e CE, em cada protocolo experimental, são apresentados nas Tabelas 1 e 2, nesta ordem.

Tabela 1: Somatório das repetições máximas realizadas nas três séries no exercício leg press

\begin{tabular}{ccccc}
\hline Protocolos & PSA & PSI & P10 & P20 \\
\hline Média e DP & $23 \pm 1$ & $17 \pm 2^{*}$ & $18 \pm 1^{*}$ & $20 \pm 1^{*} \dagger \ddagger$
\end{tabular}

DP: Desvio-padrão; PSA: protocolo sem alongamento prévio; PSI: protocolo sem intervalo entre AEP e TF; P10: intervalo de dez minutos entre AEP e TF; P10: intervalo de 20 minutos entre AEP e TF. *diferenças significativas quando comparado a PSA; tdiferenças significativas quando comparado a PSI; ‡ diferenças significativas quando comparado P10.

Quanto ao exercício LP (Tabela 1), foi observada diferença significativa no número de repetições entre os protocolos $(p=0,001 ; F=$ 1786,5). Também foi observada redução significante no número total de repetições máximas completadas após o AEP nos protocolos PSI $(17 \pm 2 ; p=0,001), \mathrm{P} 10(18 \pm 1 ; p=0,02)$ e P20 $(20 \pm 1 ; p=0,004)$, comparados ao PSA $(23 \pm 1)$. Adicionalmente, no P20, verificou-se diferença significativa para o PSI $(p=0,001)$, bem como para o P20 em relação ao P10 $(p=0,023)$.

Em relação ao exercício $C E$, também foi observada diferença significativa entre os protoco$\operatorname{los}(p=0,001 ; F=1544,2)$. Na Tabela 2 nota-se que, de um modo geral, ocorreu uma redução significativa no número total de repetições máximas completadas após o AEP nos protocolos PSI $(18 \pm 1 ; p=0,001)$ e P10 $(19 \pm 2 ; p=0,021)$ comparados ao PSA $(23 \pm 1)$ no exercício CE. No P20 $(22 \pm 2 ; p=0,001)$ verificou-se diferença signifi- 
Tabela 2: Somatório das repetições máximas realizadas nas três séries na cadeira extensora

\begin{tabular}{ccccc}
\hline Protocolos & PSA & PSI & P10 & P20 \\
\hline Média e DP & $23 \pm 1$ & $18 \pm 1^{*}$ & $19 \pm 2^{*}$ & $22 \pm 2 \dagger$ \\
\hline
\end{tabular}

DP: Desvio-padrão; PSA: protocolo sem alongamento prévio; PSI: protocolo sem intervalo entre AEP e TF; P10: intervalo de 10 minutos entre AEP e TF; P10: intervalo de 20 minutos entre AEP e TF. *Diferenças significativas quando comparado a PSA; †Diferenças significativas quando comparado a PSI.

cativa para o PSI. Por outro lado, após intervalos de 20 minutos (P20) não ocorreu decréscimo significativo no desempenho de repetições quando comparado ao PSA.

\section{Discussão}

O principal achado deste estudo foi a redução significativa no total de repetições máximas realizadas nos protocolos sem intervalo (PSI), após 10 minutos (P10) e 20 minutos (P20) de intervalo entre AEP no quadríceps e TF, quando comparados ao protocolo sem alongamento (PSA) no exercício LP. Quanto ao exercício de $\mathrm{CE}$, não houve diferença significativa em relação ao P20, comparado ao PSA, exceto para os protocolos PSI e P10, que apresentaram redução significativa no número de repetições realizadas para o protocolo sem AEP prévio. Alguns autores identificaram efeitos negativos do AEP sobre a força muscular ${ }^{8,9,16-20}$, enquanto outros demonstram não haver influência ${ }^{10,21}$. Entretanto, neste trabalho, objetivou-se verificar a influência de diferentes intervalos de recuperação entre AEP de quadríceps em uma sessão de TF para exercícios multiarticulares (LP) e monoarticulares, considerando a lacuna existente em relação a tal condição.

Em linhas gerais, os resultados observados corroboram os estudos prévios que verificaram efeito deletério agudo no desempenho muscular após a realização do $\mathrm{AEP}^{11,12,14,16,17}$. Entretanto, observou-se uma tendência na redução no desempenho de repetições máximas imediata- mente após o AEP (PSI), bem como posterior aos intervalos de 10 e 20 minutos no exercício LP. Já no exercício de CE, não houve diferença significativa no desempenho de repetições máximas entre P20 e PSI. Em ambos os exercícios, verificou-se aumento na capacidade de produzir repetições com o aumento progressivo do tempo de intervalo de recuperação entre os estímulos (AEP e TF). Em relação ao desempenho de repetições executadas, a diferença entre os exercícios LP e CE pode estar associada à sobrecarga e à quantidade de massa muscular envolvida, consideravelmente maior no LP, em comparação ao exercício CE. Neste sentido, o intervalo de 20 minutos possibilitou uma completa recuperação e evitou o efeito deletério induzido pelo AEP no quadríceps durante a realização da CE.

Behm et al. ${ }^{17}$ e Power et al. ${ }^{19}$ observaram um decréscimo no desempenho muscular dos extensores de joelho após a realização do AEP. Behm et al. ${ }^{17}$ também verificaram que regimes prolongados de AEP podem inibir a CVM na ativação do músculo extensor do joelho. Considerando esse aspecto, dois principais mecanismos têm sido utilizados para indicar a redução de força induzida pelo alongamento, são elas: (a) modificações estruturais na unidade musculotendínea e, (b) fatores neurais, como a diminuição na excitabilidade dos motoneurônios, que pode reduzir a ativação muscular ${ }^{11}$. Kubo et al..$^{22}$ afirmam que há possibilidade de que os exercícios de alongamento sejam capazes de alterar as propriedades viscoelásticas da unidade músculo-tendão, reduzindo a tensão passiva e a rigidez da unidade. Egan et al. ${ }^{10}$ citam que o AEP pode agir sobre o órgão tendinoso de Golgi, acarretando a liberação do neuromediador inibitório gama-aminobutírico na medula, provocando assim a diminuição da força. Todavia, Wilson et al. ${ }^{23}$ citam que um sistema músculo-tendão mais maleável passaria por um rápido período de diminuição de comprimento, com ausência de sobrecarga, até que os componentes elásticos do sistema fossem ajustados o suficiente para a transmissão de força, colocando o componente contrátil em uma posição menos favorável em termos de 
produção de força. Dessa forma, o intervalos de recuperação superiores a 20 minutos parecem ser adequados para a realização do treinamento concorrente de flexibilidade e força sem induzir efeitos deletérios sobre o desempenho muscular, considerando os resultados observados neste estudo. Entretanto, pode-se perceber que fatores como tipo de exercício, número de grupamentos musculares envolvidos, quantidade de massa muscular podem influenciar nesse tempo de recuperação.

Corroborando os achados deste estudo, Arruda et al. ${ }^{20}$ observaram as respostas agudas de dois protocolos de aquecimento previamente ao teste de 10RM - um grupo utilizou o aquecimento específico; e o outro se aqueceu por meio de exercícios de alongamento. Observando os resultados, os efeitos dos exercícios de alongamento se mostraram negativos em relação ao teste de força, pois houve um decréscimo no número de repetições máximas, assim como foi encontrado neste trabalho. No estudo de Tricoli e Paulo $^{18}$, observou-se uma diminuição no teste de 1RM para os exercícios de extensão e flexão de joelho após realização de AEP. Com resultados semelhantes, Endlich et al. ${ }^{8}$ encontraram uma redução significativa na carga máxima alcançada no teste de 10RM, em membros superiores, quando precedido por sessões longas (maiores que oito minutos) de alongamento; já para membros inferiores, ambas as condições experimentais com alongamento foram capazes de reduzir significativamente a força no teste de 10RM. Concluiu-se que sessões de AEP efetuadas antes de atividades que envolvam força dinâmica possuem a capacidade de alterar negativamente o desempenho dessa qualidade física, acarretando pior rendimento após volumes elevados de alongamento muscular. Resultados semelhantes foram comentados por Gurjão et al. ${ }^{9}$, que avaliaram o efeito agudo do AEP na ativação muscular e produção de força e verificaram que a capacidade de produção de força muscular diminuiu após a execução do alongamento estático.

Egan et al. ${ }^{10}$ avaliaram os efeitos agudos do AEP sobre o pico de torque e a potência mé- dia durante a contração voluntária máxima dos extensores de joelho em jogadoras de basquete. Assim como neste estudo, foram adotados diferentes intervalos $(5,15,30$ e 45 minutos) entre o alongamento muscular e o TF. Diferente dos resultados aqui mostrados, os achados indicaram que o AEP não teve impacto nas medidas avaliadas. Entretanto, esses resultados podem estar relacionados ao nível de treinamento das participantes, atletas de basquete, diferente da amostra utilizada nesta pesquisa, que foi composta por jovens praticantes de TF.

Em relação ao efeito do volume do AEP sobre a produção de força muscular, Behm, Button e Butt ${ }^{17}$ aplicaram AEP no quadríceps durante 15 a $20 \mathrm{~min}$, enquanto Fowles, Sale e MacDougall1 ${ }^{11}$ alongaram os músculos extensores do tornozelo por $30 \mathrm{~min}$, resultando em redução na força e ativação muscular. Tais achados sugerem que a duração dos efeitos deletérios promovidos pela aplicação do AEP sobre o desempenho muscular em testes de força sofre interferência direta de fatores, tais como número de exercícios de alongamento e repetições aplicados, associando-se, dessa forma, a possíveis alterações neurais e mecânicas que afetam a produção de força muscular.

Fowles, Sale e MacDougall ${ }^{11}$ concluíram em seu estudo que a diminuição da força muscular estaria associada à redução no recrutamento de unidades motoras, ativação dos órgãos tendinosos de Golgi e contribuição dos nociceptores. Para McBride, Diane e Nimphius ${ }^{12}$, o tempo prolongado de alongamento determina acomodação das fibras, de forma a comprometer a transmissão de mensagens motoras, ocasionando deformação nos componentes plásticos musculares e redução do tônus muscular. Adicionalmente aos mecanismos neurais, questões mecânicas estariam envolvidas nesses achados, pois modificações na relação comprimento-tensão com alteração da sobreposição fisiológica entre os filamentos de actina e miosina já foram demonstradas ${ }^{10}$.

Destaca-se que nos estudos supracitados não foi especificado se os indivíduos realizavam treinamento de flexibilidade com frequência, bem como se houve familiarização com os 
exercícios realizados. De acordo com Sandberg et al. ${ }^{24}$, os níveis prévios de flexibilidade dos indivíduos podem interferir de maneira significativa na produção de força muscular em resposta ao exercício de alongamento aplicado por intermédio do ponto de disparo do fuso muscular. Neste sentido, sujeitos que praticam treinamento de flexibilidade com frequência apresentam menor suscetibilidade a alterações plásticas agudas após exercícios de alongamento ${ }^{25}$.

Em relação ao tempo de intervalo entre AEP e TF, Figueiredo et al. ${ }^{26}$ observaram redução significativa no efeito inibitório promovido pelo alongamento estático sobre o número de repetições máximas realizadas no exercício supino reto com cargas de 10RM e intervalo de três minutos entre as séries (3), após intervalos de dez minutos entre os exercícios supracitados. De acordo com os autores, o volume moderado de AEP (três séries de dez segundos para o peitoral maior, deltoide e tríceps) possibilitou rápida recuperação e manutenção do desempenho muscular comparado à condição sem alongamento muscular.

Destaca-se que uma das limitações deste estudo associa-se a aplicação dos protocolos apenas com exercícios para um grupo muscular (quadríceps). Haja vista que em uma sessão de TF tradicional são realizadas séries múltiplas para diversos exercícios e grupos musculares. Apesar disso, os achados apresentados são importantes, pois o protocolo adotado está diretamente relacionado à realidade prática de treinadores e praticantes de TF durante a elaboração de programas de exercícios físicos. Adicionalmente, são escassos na literatura estudos que investigaram a influência do intervalo de recuperação entre protocolos de AEP e desempenho muscular em testes de força aplicados em exercícios convencionalmente realizados em academias e centros de treinamento. Considerando que praticantes de TF e atletas realizam o treinamento de flexibilidade e TF na mesma sessão de treinamento, tais achados reforçam, assim, a necessidade de compreender a influência de ambos os estímulos sobre os níveis de força muscular.

\section{Conclusão}

Conforme os resultados obtidos no estudo, pode-se concluir que AEP aplicado antes do TF, nos exercícios LP e CE, reduziram a quantidade de repetições máximas realizadas de forma aguda. Entretanto, ao adotar intervalos de até 20 minutos entre o AEP e a execução dos exercícios, foi verificado que nos menores intervalos de recuperação, ocorreu uma maior diminuição do número de repetições nos exercícios e, no intervalo de 20 minutos, não se observou diferença significativa entre P20 e PSA no exercício CE. Logo, intervalos superiores a 20 minutos parecem ser adequados para minimizar o efeito deletério do AEP sobre o desempenho de repetições completadas em exercícios monoarticulares para membro inferior, enquanto intervalos inferiores a esse tempo parecem influenciar de forma negativa na força muscular.

\section{Agradecimentos}

Dr. Humberto Miranda agradece à Fundação de Amparo à Pesquisa do Estado do Rio de Janeiro (FAPERJ), e o Prof. Gabriel Paz agradece à Coordenação de Aperfeiçoamento Pessoal de Nível Superior (CAPES) e ao Programa de Educação pelo Trabalho e Saúde (PET-Saúde).

\section{Referências}

1. Fletcher GF, Balady G, Froelicher VF, Hartley LH, Haskell WL, Pollock ML. Exercise Standards. A statement for healthcare professionals from the American Heart Association Writing Group. Circulation. 1995;91:580-615.

2. American College of Sports Medicine. ACSM position stand: progression models in resistance training for healthy adults. Med Sci Sports Exer. 2009;41:687-708. 
3. American College of Sports Medicine. ACSM position stand: quantity and quality of exercise for developing and maintaining cardiorespiratory, musculoskeletal, and neuromotor fitness in apparently healthy adults: guidance for prescribing exercise. Med Sci Sports Exerc. 2011;43:1334-59.

4. Ribeiro SG. O alongamento como fator interveniente na hipertrofia muscular: um estudo preliminar. Rev Conexões. 2008;6(1):35-46.

5. Viveiros LE, Simão R. Treinamento da flexibilidade: uma abordagem metodológica. Rev Baiana de Educ Fis. 2001;2:20-6.

6. Achour Júnior A. Exercícios de alongamento: anatomia e fisiologia. Barueri: Manole; 2002.

7. Silva GVLC, Silveira ALB, Di Masi F, Bentes CM, Miranda HL, Novaes JS. Efeito agudo do alongamento estático sobre a força muscular isométrica. ConScientiae Saúde. 2012;11(2):274-80.

8. Endlich PW, Farina GR, Dambroz C, Gonçalves WLS, Moysés MR, Mill JG, Abreu GR. Efeitos agudos do alongamento estático no desempenho da força dinâmica em homens jovens. Rev Bras Med Esporte. 2009;15:200-3.

9. Gurjão ALD, Gonçalves R, de Moura RF, Gobbi $\mathrm{S}$. Acute effect of static stretching on rate of force development and maximal voluntary contraction in older women. J Strength Cond Res. 2009;23:2149-54.

10. Egan AD, Cramer JT, Massey LL, Marek SM. Acute effects of static stretching on peak torque and mean power output in National Collegiate Athletic Association Division I women's basketball players. J Strength Cond Res. 2006;20(4):778-82.

11. Fowles JR, Sale DG, MacDougall JD. Reduced strength after passive stretch of the human plantar flexors. J Appl Physiol. 2000;89:1179-88.

12. McBride JM, Deane R, Nimphius N. Effect of stretching on agonist-antagonist muscle activity and muscle force output during single and multiple joint isometric contractions. Scand J Med Sci Sports. 2007;17(1):54-60.

13. Paz GA, Maia MF, Lima VP, Oliveira CG, Bezerra E, Simão R, Miranda H. Maximal exercise performance and electromyography responses after antagonist neuromuscular proprioceptive facilitation: a pilot study. JEPonline. 2012;15(6):60-7.

14. Chaves CPG, Guerra CPC, Moura SRG, Nicoli AIV, Félix I, Simão R. Déficit bilateral nos movimentos de flexão e extensão de perna e flexão do cotovelo. Rev Bras Med Esporte. 2004;10:505-8.
15. Miranda H, Simao R, dos Santos Vigário P, de Salles BF, Pacheco MT, Willardson JM. Exercise order interacts with rest interval during upperbody resistance exercise. J Strength Cond Res. 2010;24(6):1573-7.

16. Lopes RSD, Barja PR, de Matos LKBL, Delmondes FF, Lopes PFD, Silva KAS, Lima MO. O efeito do tempo de duas diferentes técnicas de alongamento na amplitude de movimento. ConScientiae Saúde. 2010;9(4):603-9.

17. Behm DG, Button DC, Butt JC. Factors affecting force loss with prolonged stretching. Can J Appl Physiol. 2001;26: 261-72.

18. Tricoli V, Paulo AC. Efeito agudo dos exercícios de alongamento sobre o desempenho de força máxima. Rev Bras Ativ Fís Saúde. 2002;7:6-13.

19. Power K, Behm D, Cahill F, Carrol M, Young W. An acute bout of static stretching: effects on force and jumping performance. Med Sci Sport Exer. 2004;36:1389-96.

20. Arruda FLB, Faria LB, Silva V, Senna GW, Simão R, Novaes J, Maior AS. A influência do alongamento no rendimento do treinamento de força. Rev Treinamento Desportivo. 2006;7:1-5.

21. Silveira RN, Farias JM, Alvarez BR, Bif R, Vieira J. Efeito agudo do alongamento estático em músculo agonista nos níveis de ativação e no desempenho da força de homens treinados. Rev Bras Med Esporte. 2011;17:26-30.

22. Kubo K, Kanehisa H, Kawaakami Y, Fukunaka $\mathrm{T}$. Influence of static stretching on viscoelastic properties of human tendon structures in vivo. J Appl Phys. 2001;90:520-7.

23. Wilson GJ, Murphy AJ, Pryor JF. Muscle tendinous stiffness: its relationship to eccentric, isometric, and concentric performance. J Appl Phys. 1994;76:2714-9.

24. Sandberg JB, Wagner DR, Willardson JM, Smith GA. Acute effects of antagonist stretching on jump height, torque and electromyography of agonist musculature. J Strength Cond Res. 2012;26(5):1249-56.

25. Costa e Silva GVL, Silveira ALB, Mazi F, Bentes CM, Miranda H, Novaes JS. Efeito agudo do alongamento estático sobre a força muscular isométrica. ConScientiae Saúde (Impresso). 2012;11:274-80.

26. Figueiredo T, Andrade RSJ, Oliveira MS, Bertoldo TR, Simão R, Miranda H. Influência de diferentes intervalos entre o alongamento e uma sessão de treinamento de força. Terapia Manual. 2011;9:343-7. 